\title{
An Experimental Study on Oxidation Rate of Coal:The Influence of Oxygen Concentration
}

\author{
Bucai Ma \\ School of Resource and Safety Engineering \\ China University of Mining and Technology (Beijing) \\ Beijing, China \\ liuwei7230@qq.com \\ Youqiang Chen \\ School of Resource and Safety Engineering \\ China University of Mining and Technology (Beijing) \\ Beijing, China \\ 15650712420@163.com
}

\author{
Yinlei Yang \\ School of Resource and Safety Engineering \\ China University of Mining and Technology (Beijing) \\ Beijing, China \\ 1134189566@qq.com \\ Ying Zhu \\ School of Resource and Safety Engineering \\ China University of Mining and Technology (Beijing) \\ Beijing, China \\ 867163588@qq.com
}

\begin{abstract}
In order to investigate the oxidation characteristics of residual coal of goaf under different oxygen concentration environment, the discarded coal is collected from the actual workplace to analyze the particle size distribution, then five coal samples are prepared by the same granularity ratio. The heating and oxidizing experiments of the coal samples are conducted, on the inlet oxygen concentration at $1.9 \%, 6.0 \%, 10.3 \%, 15.1 \%$ and $20.0 \%$ respectively, to detect the outlet oxygen concentration in the heating process. Supported by the experimental data, the formulas of the standard oxygen consumption rate are deduced respectively to obtain the change law of the oxidation rate of each coal sample. The results show that the standard oxygen consumption rates are basically equal under different inlet oxygen concentration at the same temperature, which verifies the correctness of the coaloxygen compound theory. What's more, the curve of the standard oxygen consumption rate with the temperature rising can be divided as the slow oxidation phase, the accelerated oxidation phase and the violent oxidation phase in sequence. The results of the research have a theoretical and practical significance for preventing the spontaneous fire of coal in goaf.
\end{abstract}

Keywords-spontaneous combustion; particle size distribution; oxygen concentration; residual coal; oxygen consumption rate

\section{INTRODUCTION}

Oxygen plays an important role in the self-heating process of coal [1]. In low temperature oxidation stage of coal, according to the coal and oxygen compound theory, the physical absorption, the chemical adsorption and the chemical reaction in sequence [2,3] take place between the active structure on the coal surface and oxygen in the air, and a lot of heat is released. When the oxygen supplies sufficiently and the heat storage conditions are quite good, the heat from the coal oxidation reaction will accumulate to make the coal temperature rising, resulting in spontaneous combustion eventually at coal stockpiles, transportation over the long distances, or underground mining.

As well known, the spontaneous combustion is one of the most serious disasters in the coal mine [4,5]. Statistics show that, in China, more than $60 \%$ of the spontaneous combustion underground happen in goaf, which threatened the normal production of workplace, and easily caused gas explosion in the goaf of gassy mine, resulting in heavy casualties. On March 29, 2013, gas explosion occurred in Babao Coal Mine in Jilin province and 28 people were killed, the reason for which was spontaneous combustion in the goaf. Therefore, oxygen as one of the main influence factors for the coal spontaneous heating should be studied, which is of great significance for preventing the spontaneous fire.

\section{EXPERIMENTAL APPARATUS AND PROCESS}

At present, the low temperature oxidation experimental systems of coal mainly include two types: programmed temperature $[6,7,8]$ and adiabatic oxidation $[9,10]$, but both of them use the air bath to heat up the coal sample tank. Because the thermal conductivity of air is poor, the temperature in the air bath heating cabinet is not equal everywhere, which causes the coal sample tank to heat unevenly and then the temperature of each point in tank is different. This brings a lot of problems, such as the oxygen consumption rate calculation of coal. The oxygen consumption rate is affected by temperature and oxygen concentration at the same time, only when the requirement of temperature equality in coal sample tank is met everywhere, the effects of temperature should be eliminated, so the oxygen consumption could be regard as a linear distribution along the axial of coal sample tank and then the total oxygen consumption of the whole tank per unit time could be obtained by linear integral. If the temperature differences in the tank are large, the oxygen consumption cannot be accurately calculated.

Therefore, the low temperature oxidation experimental system of coal with oil bath is developed independently, as 
shown in Fig.1. The specific heat and the thermal conductivity of the silicone oil are bigger than that of air, so the silicone oil is used as the heat transfer medium in the experimental system. And the coal sample tank is made into a slender cylindrical $(25 \mathrm{~mm} \times 300 \mathrm{~mm})$. These two main improvements make the coal sample tank be heated more uniformly.

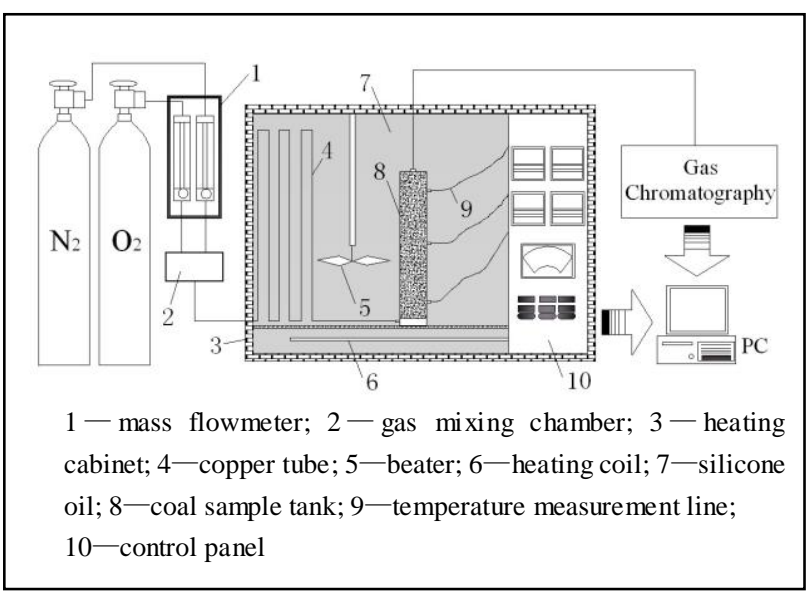

Figure 1. Experimental device structur

The experiment system includes: 1) the gas supply system, made up of gas cylinders, mass flowmeter and gas mixing chamber; 2) the heating and constant temperature system, composed of coal sample tank, heating cabinet, silicone oil and program temperature control table; 3) gas chromatography; 4) the temperature data acquisition system.

In the experiment, the coal sample is put into the sample tank firstly and then the nitrogen gas is injected. After the coal temperature stabilized, a certain concentration of oxygen proportioned by the mass flowmeter, instead of the nitrogen, is injected at inflow rate of $60 \mathrm{ml} / \mathrm{min}$ and is maintained throughout the experiment. Then the tank is heated, i.e., the oil temperature is rising up $15^{\circ} \mathrm{C}$ at the rate of $1{ }^{\circ} \mathrm{C} / \mathrm{min}$ and then the oil temperature kept constant for $40 \mathrm{~min}$. In the temperature constant period, the outlet gas of the tank began to be collected when the coal temperature is stabilized, namely the rise range is less than $0.5{ }^{\circ} \mathrm{C}$ within $5 \mathrm{~min}$. The gas chromatograph is used to detect the outlet oxygen concentration. This ensured that the oxygen concentration at the coal temperature stabilized period is obtained. And then continued to heat up, repeated the process until the coal temperature arrived at $200^{\circ} \mathrm{C}$.

In order to analyze the temperature changes of different location in coal sample tank within the heating process, three thermocouple probes are equipped in turn at the top, middle and bottom of the tank. The temperature changes of the three probes for 2\#-5\# coal sample from the heating beginning to the end are showed in Fig.2. As it can be seen, in the experiment process, the temperature change curves of the three probes of each coal sample are basically in coincidence. It shows that the oil bath type experimental system should basically guarantee the temperature everywhere in the tank uniform. In addition, the temperature of $2 \#-5 \#$ coal sample from room temperature to $200^{\circ} \mathrm{C}$ spent $840,820,740$ and $720 \mathrm{~min}$ respectively. It indicates that the lower the oxygen concentration is, the smaller the exothermic oxidation of coal is, so more time is spent for the temperature rise. In Fig .2, the temperature curves appeared as a stepladder increase. This is because the heating is a repeated process of the temperature rising and keeping constant.

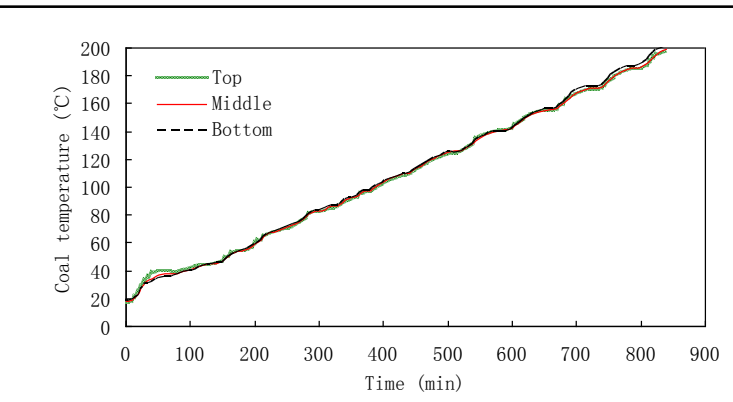

(a) $2 \#(6.0 \%)$

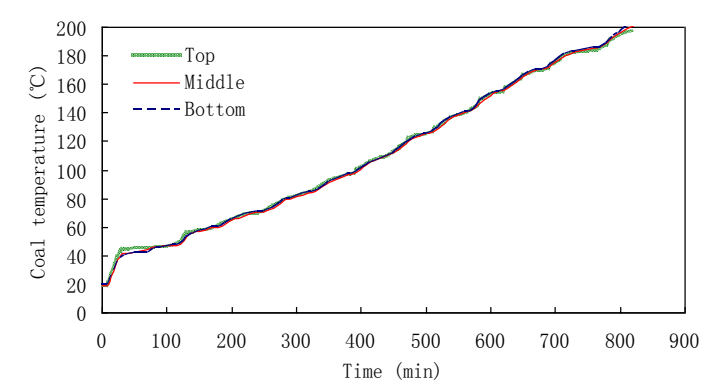

(b) $3 \#(10.3 \%)$

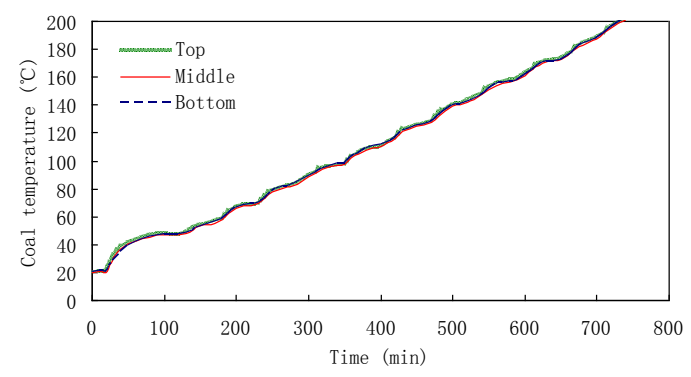

(c) $4 \#(10.3 \%)$

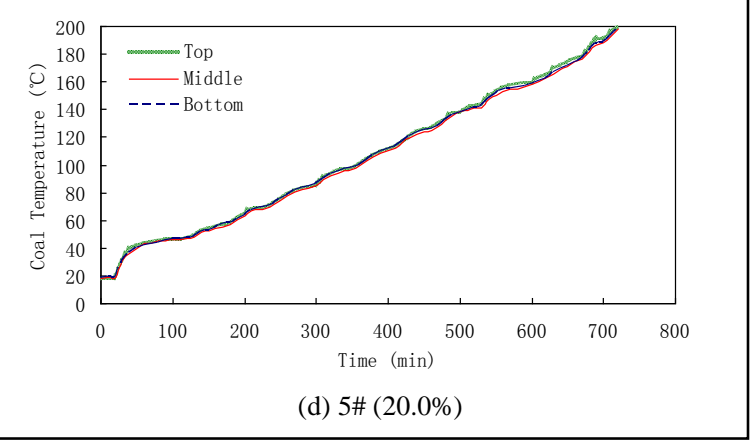

Figure 2. Curves of the coal temperature rising

\section{EXPERIMENTAL RESULTS}

Under different inlet oxygen concentration, the outlet oxygen concentration of the tank is analyzed, and plotted in Fig.3.

It can be obtained from Fig.3:

The outlet oxygen concentration of each coal sample gradually reduces with the temperature rising. When the coal temperature is under $140^{\circ} \mathrm{C}$, the reducing is slow, but 
when exceeding $140^{\circ} \mathrm{C}$, the oxygen concentration sharply drops.

In the same inlet oxygen concentration, the higher the coal temperature is, the less the outlet oxygen concentration is; at the same coal temperature, the higher the inlet oxygen concentration is, the higher the outlet oxygen concentration is.

The relationship between the outlet oxygen concentration and the inlet oxygen concentration is a straight linear change.

The above shows that, with the coal temperatures rise, the coal-oxygen reaction consumes more and more oxygen, and the reaction is more intense when the temperatures exceeds $140^{\circ} \mathrm{C}$.

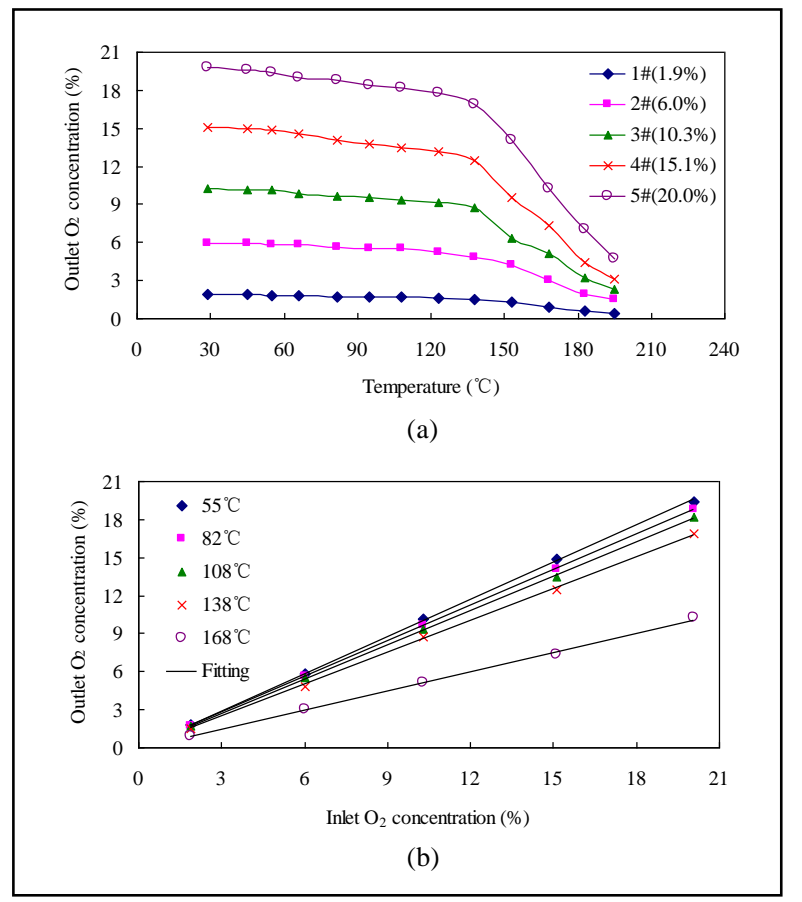

Figure 3. Curves of outlet oxygen with temperature rising

\section{ANALYSIS OF OXIDATION RATE OF COAL}

In the low temperature oxidation experiment of coal, the oxygen concentration change of each point in the crushed coal is mainly related to the air motion, the oxygen molecular diffusion and the oxygen consumption by the coal and oxygen reaction, etc. Therefore, the convection and diffusion equation of oxygen in crushed coal [11,12] is established by the mass conservation equation, expressed as

$$
\frac{\partial C}{\partial \tau}=n \cdot \operatorname{div}(k \cdot \operatorname{grad} C)-\operatorname{div}(C \cdot \vec{v})-V(t)
$$

Where, $\mathrm{C}$ is the oxygen concentration, $\mathrm{mol} / \mathrm{cm} 3 ; \tau$ is unit time, $\mathrm{s} ; \mathrm{n}$ is the void fraction, $\% ; \mathrm{k}$ is the diffusion coefficient of oxygen, $\mathrm{m} 2 / \mathrm{s} ; \vec{v}$ is the gas flow velocity in the void or porosity of coal, $\mathrm{m} / \mathrm{s} ; \mathrm{V}(\mathrm{t})$ is the real oxygen consumption rate at $\mathrm{t}$ temperature, $\mathrm{mol} /(\mathrm{cm} 3 \cdot \mathrm{s})$.

The diameter of coal sample tank is small, so is the air flow. So it can be assumed that all of the air flows along the axial direction ( $x$ direction) of the tank, and the oxygen concentration distribution equation on the axial direction is obtained as

$$
\frac{\partial C}{\partial \tau}=n \cdot \frac{\partial}{\partial x}\left(k \cdot \frac{\partial C}{\partial x}\right)-\frac{\partial(v \cdot C)}{\partial x}-V(t)
$$

Where, $\mathrm{v}$ is the air flow velocity in axial direction, $\mathrm{m} / \mathrm{s}$.

In the experiment, the rate of oxygen concentration changes with time and the oxygen concentration diffusion in crushed coal is relatively small and can be ignored to obtain the oxygen consumption rate as

$$
V(t)=-v \cdot \frac{d C}{d x}=-\frac{Q}{S \cdot n} \cdot \frac{d C}{d x}
$$

Where, $\mathrm{x}$ is axial length of the tank, $\mathrm{cm}$; $\mathrm{Q}$ is the gas flow, $\mathrm{ml} / \mathrm{min} ; \mathrm{S}$ is the sectional area of tank, $\mathrm{cm}^{2}$.

The coal-oxygen compound theory [13], the most widely accepted cause theory of spontaneous combustion at present, holds that the heat released by chemical adsorption, chemical reaction between coal surface and oxygen is the root cause for the coal temperature rising $[14,15]$. The oxidation reaction of coal will be accelerated by the increased temperature to consume more oxygen and release more heat. If the heat production is sustained more than the heat dissipation in the environment, the coal temperature will be rising and the spontaneous combustion will happen as a result. According to the theory, the oxygen consumption is the direct ratio to the environmental oxygen concentration, in which the lower the oxygen concentration is in the environment, the less the oxygen is consumed, so the oxygen consumption ratio at different oxygen concentration should be the same, i.e. the ratio of inlet and outlet oxygen concentration of the tank should be the same when the inlet oxygen concentration is different. Therefore, the standard oxygen consumption rate $\mathrm{VO}(\mathrm{t})$ which is directly related to the ratio of inlet and outlet oxygen concentration is figured out to analyze the changes of the oxygen consumption when the different inlet oxygen concentration are all converted to the oxygen concentration in fresh air. From the coal-oxygen compound theory, the standard oxygen consumption rate at different oxygen concentration should be equal at the same temperature. If not, the theory should be not viable.

According to the principle of chemical kinetics, the oxygen consumption rate is proportional to the mass fraction of oxygen, expressed as equation (4). Then the standard oxygen consumption rate in fresh air (oxygen concentration is $21 \%$ ) is deduced as equation (5).

$$
V(t)=K C
$$

$$
V_{0}(t)=\frac{C_{0} \cdot V(t)}{C}
$$

Where, $\mathrm{K}$ is the chemical reaction rate constant; $\mathrm{VO}(\mathrm{t})$ is the standard oxygen consumption rate, $\mathrm{mol} /(\mathrm{cm} 3 \cdot \mathrm{s}) ; \mathrm{C} 0$ is the standard oxygen concentration, $21 \%$. 
By equation (3) and (5), the oxygen consumption $\mathrm{dC}$ of coal sample in axial length $\mathrm{dx}$ of the tank can be obtained as

$$
d C=-V_{0}(t) \cdot \frac{S \cdot n}{Q} \cdot \frac{C}{C_{0}} d x
$$

Likewise, the temperature of each point in the tank is regard as be equal. At a certain constant temperature, the integral equation is obtained in the axial length of the whole tank, as follows

$$
\int_{C_{1}}^{C_{2}} \frac{d C}{C}=-V_{0}(t) \cdot \frac{S \cdot n}{Q \cdot C_{0}} \cdot \int_{0}^{L} d x
$$

Finally, the calculation formula of standard oxygen consumption rate is obtained as equation (8), in which the $\mathrm{C} 1, \mathrm{C} 2$ are not equal to zero.

$$
V_{0}(t)=\frac{Q C_{0}}{S \cdot L \cdot n} \ln \frac{C_{1}}{C_{2}}
$$

According to equation (8), the curves of the standard oxygen consumption rate under different conditions are obtained, shown in Fig.4.

In Fig.4(a), with the coal temperature rising, the five curves of the standard oxygen consumption rate at different inlet oxygen concentration are very close. In Fig.4 (b), at the same temperature, the fitting curves of the standard oxygen consumption rate with the oxygen concentration increase are horizontal lines basically. It shows that the standard oxygen consumption rate of coal obtained in the experiment is almost equal and not affected by the size of oxygen concentration in fact. The differences of the curves in Fig.4(a) are mostly because of the measurement error in the experiment. So the correctness of the coal-oxygen compound theory is verified.

Now that the standard oxygen consumption rate is independence with the oxygen concentration, the main influence factor for that is the temperature in our experiments. By analyzing the experimental data, the curves of the standard oxygen consumption rate with the temperature rising in the whole experimental process can not be better fitted by one regression analysis function, and then two inflection points are found in all the curves, $80^{\circ} \mathrm{C}$ and $140^{\circ} \mathrm{C}$ respectively. When the temperature $<80^{\circ} \mathrm{C}$, the oxygen consumption for the oxidative and exothermic reaction of coal is small, and the rising of the curves is gently. When the temperature exceeds $80^{\circ} \mathrm{C}$, the oxidation reaction of coal begins to accelerate, and the oxygen consumption increases significantly. When exceeds $130^{\circ} \mathrm{C}$, the reaction takes place violently, and the curves of the rate rise rapidly in the straight lines. Therefore, the curves can be divided into three stages by the two points, respectively the slow oxidation phase, the accelerated oxidation phase and the violent oxidation phase, shown in the Fig.5. For the curve of each inlet oxygen concentration, the exponential function fitting is done respectively in the slow oxidation phase and the accelerated oxidation phase, and the straight line fitting is done in the violent oxidation phase. So the fitting equation of the standard oxygen consumption rate is expressed as

$$
V_{0}(t)=\left\{\begin{array}{l}
a e^{b t}\left(t<80^{\circ} C\right) \\
c e^{d t} \quad\left(80^{\circ} C \leq t \leq 140^{\circ} C\right) \\
f t+j\left(t \geq 140^{\circ} C\right)
\end{array}\right.
$$

Where, $a, b, c, d, f$ and $j$ are the coefficients of fitting, and the values are shown in Table 1.

From Table 1, at different inlet oxygen concentrations, the correlation coefficient of the fitted curve are high, which shows the three change stages divided is reasonable. The differences of the same coefficient of fitting are relatively small, which explains the five curves of the standard oxygen consumption rate are very close.

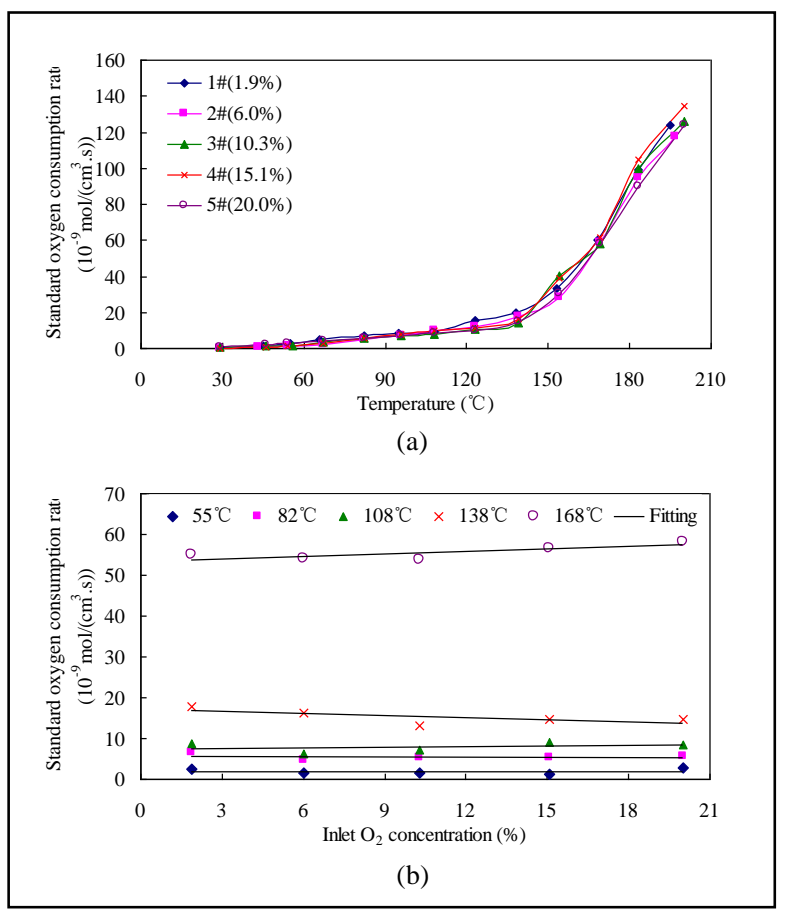

Figure 4. Curves of the standard oxygen consumption rate with temperature rising

\section{CONCLUSIONS}

The low temperature oxidation experimental system of coal with oil bath, in which the silicone oil is used as the heat transfer medium to make the coal sample tank heated more evenly, is developed independently to improve the accuracy of the experiment;

The formulas of the real and standard oxygen consumption rate are established respectively. The experimental results show that the oxygen concentration has a significant affect on the real oxygen consumption rate, in which the real oxygen consumption rate dramatically increased when the inlet oxygen concentration increased at the same temperature. But the oxygen concentration is irrelevant to the standard oxygen consumption rate, so that the correctness of the coaloxygen compound theory is verified;

This research quantitatively analyzes the influence of oxygen concentration on the oxidation rate of coal in the low temperature stage. The studies have shown that, in the same condition, the spontaneous combustion is suppressed 
with the inlet oxygen concentration reducing, and when the environmental oxygen concentration is less than $3 \%$, the coal spontaneous combustion will not happen.

TABLE I. THE COEFFICIENT OF FITTING

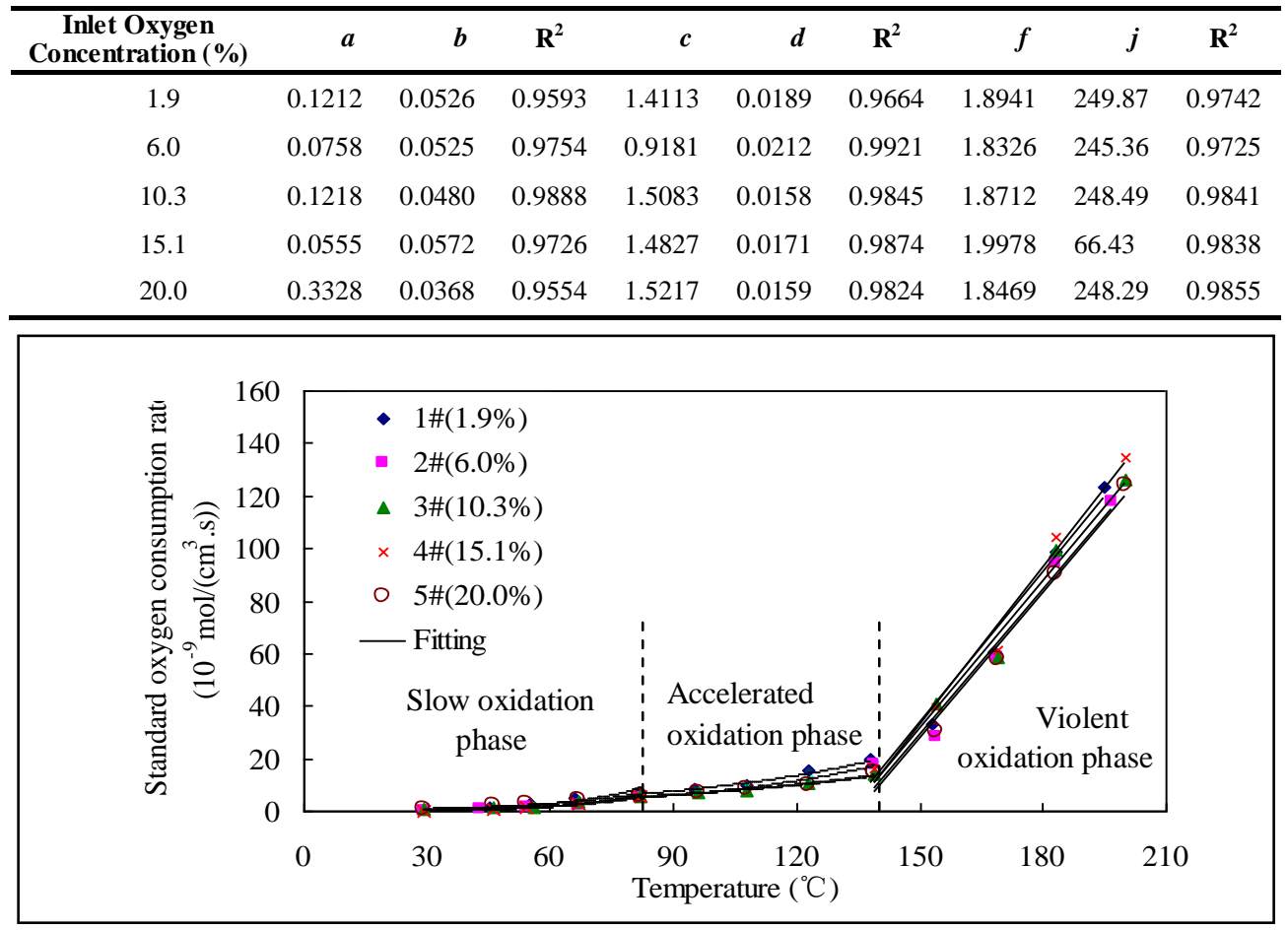

Figure 5. Fitting of the standard oxygen consumption rate with temperature rising

\section{REFERENCES}

[1] V. A. Portola, "Assessment of the Effect of Some Factors on Spontaneous Coal Combustion," Journal of Mining Science, vol. 32,1996, pp. 212-218.

[2] Wang Jiren, Deng Cunbao and Hong Lin, "Dynamics Study on Fractal Reaction of Oxygen in Granular Coal," Journal of China Coal Society, vol. 30, 2005, pp. 585-588.

[3] Deng Cunbao, Wang Jiren, and Ye Bing, "Coal Surface Physical Adsorption to Single Oxygen Molecule Mechanism," Journal of China University of Mining and Technology, vol. 38,2008, pp. 171-175.

[4] B. Taraba, and Z Michalec, "Effect of Longwall Face Advance Rate on Spontaneous Heating Process in the Gob Area - CFD Modeling," Fuel, vol. 90, 2011, pp. 2790-2797.

[5] Xie Jun, Xue Sheng, and Cheng Weimin, "Early Detection of Spontaneous Combustion of Coal in Underground Coal Mines with Development of an Ethylene Enriching System," International Journal of Coal Geology, vol. 85, 2011, pp. 123-127.

[6] Zhong Xiaoxing, Wang Deming, and Yi Xiaodan, "Test Method of Critical Temperature of Coal Spontaneous Combustion Based on the Temperature Programmed Experiment," Journal of China Coal Society, vol. 35(sup), 2010, pp. 128 - 131. (in chinese)

[7] W. Sujanti, and Zhang Dongke, "A Laboratory Study of Spontaneous Combustion of Coal: the Influence of Inorganic Matter and Reactor Size" Fuel, vol. 78, 1999, pp. 549-556.
[8] Qi Xuyao, Wang Deming, and Zhong Xiaoxing, "Characteristics of Oxygen Consumption of Coal at Programmed Temperatures," Mining Science and Technology, vol. 20, 2010, pp. 0372-0377.

[9] T. X. Ren, J.S. Edwards, and D. Clarke, "Adiabatic Oxidation Study on the Propensity of Pulverised Coals to Spontaneous Combustion," Fuel,vol. 78, 1999, pp. 1611-1620.

[10] B. B. Beamish, M. A. Barakat, and J. D. St. George, "Spontaneous-combustion Propensity of New Zealand Coals under Adiabatic Conditions," International Journal of Coal Geology, vol. 45, 2001, pp. 217-224.

[11] S. Krishnaswamy, P. K. Agarwal and R. D. Gunn, "Lowtemperature Oxidation of Coal 3. Modelling Spontaneous Combustion in Coal Stockpiles,” Fuel, vol. 75, 1996, pp. 353 362.

[12] H. Wang, B. Z. Dlugogorski, and E. M. Kennedy, "Theoretical Analysis of Reaction Regimes in Low-Temperature Oxidation of Coal," Fuel, vol. 78, 1999, pp. 1073-1081.

[13] Wang Jiren, and Sun Yanqiu, "Basic Theory Research of Coal Spontaneous Combustion," Journal of Coal Science and Engineering, vol. 14, 2008, pp. 239-243

[14] Wang Haihui, B. Z. Dlugogorski, and E. M. Kennedy, "Coal Oxidation at Low Temperatures: Oxygen Consumption, Oxidation Products, Reaction Mechanism and Kinetic Modeling. Progress in Energy and Combustion," Science, vol. 29, 2003, pp. 487-513.

[15] J. S. Gethner, "Thermal and Oxidation Chemistry of Coal at Low Temperatures," Fuel, vol. 64, 1985, pp. 1443-1446. 